\title{
Bem-estar subjetivo: autoavaliação em estudantes universitários
}

\author{
Subjective well-being: self-assesment \\ in college students
}

\author{
Paola PASSARELI-CARRAZZONI ${ }^{1}$ \\ José Aparecido DA SILVA²
}

\begin{abstract}
Resumo
O bem-estar subjetivo surgiu como um interessante tema da Psicologia Positiva e tem recebido cada vez mais atenção de pesquisadores da área. Este estudo teve como objetivo principal verificar a eficácia de três instrumentos de autoavaliação do bem-estar subjetivo, a partir de uma população de estudantes universitários da cidade de Ribeirão Preto. Foi realizada a análise estatística descritiva e a análise de componentes principais para os resultados apresentados no Questionário de Felicidade Oxford e na Escala de Felicidade Subjetiva. O Item de Felicidade Global foi analisado apenas por intermédio da estatística descritiva, pois apresentava uma única questão. Partindo do fato de que pesquisas realizadas indicam uma diferença pouco significativa entre autoavaliações realizadas por homens e por mulheres, este estudo traz resultados que confirmam essa constatação quanto às medianas apresentadas em todos os instrumentos, porém aponta diferenças significativas em relação à estrutura dos instrumentos de homens e mulheres para o Questionário de Felicidade Oxford.
\end{abstract}

Unitermos: Autoavaliação. Bem-estar. Estudantes universitários.

\begin{abstract}
Subjective well-being has appeared as an interesting new subject of Positive Psychology, and as such, has received increasing attention from researchers of the area. In this study the aim was to verify the effectiveness of three self-report instruments of subjective well-being in a population of college students in Ribeirão Preto. The data was submitted to descriptive statistics analysis and to Principal Component Analysis of the results presented in the Oxford Happiness Questionnaire and the Subjective Happiness Scale. Both the instruments presented Cronbach's alpha values adjusted for men and women's results, and for all groups. The Global Happiness Item was analyzed only by means of descriptive statistics, because there was only one item presented. Previous researches have indicated no significant difference between auto-evaluations made by men and women. The results of the present study indicate that this confirmed as regards the medians presented in all the instruments, however, they point out significant differences are presented as regards the structure of the instruments of men and women for the Oxford Happiness Questionnaire.
\end{abstract}

Uniterms: Self-evaluation. Well-Being. College students.

- Universidade de São Paulo, Faculdade de Filosofia Ciências e Letras de Ribeirão Preto, Programa de Pós-Graduação em Psicobiologia. Av. Bandeirantes, 3900, Bloco 6, Monte Alegre, 14040-90, Ribeirão Preto, SP, Brasil. Correspondência para/Correspondence to: P. PASSARELI-CARRAZZONI. E-mail: <paolapc@pg.ffclrp.usp.br>.

2 Universidade de São Paulo, Faculdade de Filosofia Ciências e Letras de Ribeirão Preto, Departamento de Psicologia e Educação. Ribeirão Preto, SP, Brasil. 
Cada vez mais as pessoas têm voltado sua atenção para o estudo da Psicologia Positiva. Seligman e Csikszentmihalyi (2000, p.5) afirmam que esse campo da Psicologia está situado na avaliação de experiências subjetivas como o "bem-estar, contentamento e satisfação (no passado), esperança e otimismo (em relação ao futuro), e felicidade (no momento presente)".

Reconhecido como importante componente da Psicologia Positiva, o bem-estar subjetivo - também chamado de felicidade (Diener, 2000; Seligman, 2004) - é definido como uma ampla categoria que inclui respostas emocionais das pessoas, satisfações dominantes e julgamento global de satisfação de vida (Diener, Suh, Lucas \& Smith, 1999). Considera-se o bem-estar subjetivo como uma área ampla, e não como um constructo único. Por meio da evolução nos estudos realizados, atualmente considera-se que o bem-estar subjetivo tem um número de componentes separados, como a satisfação de vida, o afeto positivo e os baixos níveis de afeto negativo (Diener, 2000).

Muitos estudos têm sido realizados com o objetivo de ampliar os conhecimentos sobre o tema. Uma extensa revisão bibliográfica (Passareli \& Da Silva, 2007) acerca do início da Psicologia Positiva e do estudo bem-estar subjetivo apoia a consideração de que os estudos envolvendo o bem-estar subjetivo têm grande importância no entendimento do ser humano a partir de suas potencialidades, de suas forças e de suas virtudes.

\section{O bem-estar subjetivo e seus correlatos}

Fredrickson (1998) indica que as emoções positivas podem fortalecer o repertório momentâneo de pensamentos e ações individuais e, assim, auxiliar na construção de recursos pessoais permanentes, incluindo recursos físicos, intelectuais e sociais. Ela afirma que essa teoria do fortalecimento explicaria por que as emoções positivas podem promover a saúde e o bem-estar.

Em seu estudo, Diener e Seligman (2002) sugerem que as pessoas do grupo de indivíduos muito felizes experimentam sentimentos positivos a maior parte do tempo, e relatam apenas humores negativos ocasionais. Os autores sugerem que pessoas muito felizes têm um funcionamento do sistema de emoções que favorece a reação apropriada diante dos diferentes eventos de vida.
Outro importante correlato do bem-estar subjetivo são os aspectos biológicos. Segundo Salovey, Rothman, Detweiler e Steward (2000), estados emocionais positivos podem promover a crença na percepção de saúde e o próprio bem-estar físico. Steptoe, Wardle e Marmot (2005) apresentam evidências de que o afeto positivo estaria associado à boa saúde física. Segundo Steptoe et al. (2005), a relação entre redução de cortisol e afeto positivo é potencialmente relevante para a saúde. Os estados de afeto positivo são relacionados a perfis favoráveis de funcionamento em diversos sistemas biológicos e podem, através disso, ser relevantes ao reduzirem o risco de desenvolvimento de doenças físicas (Steptoe et al., 2005).

Além disso, os estados emocionais estariam relacionados à maneira como pessoas que já tenham sua saúde comprometida encaram suas dificuldades. Contrariando a opinião popular de que pessoas com menos saúde seriam menos felizes do que aquelas que apresentam melhores condições de saúde, estudos recentes indicam que pacientes em hemodiálise são tão felizes quanto pessoas saudáveis, além de estimarem sua felicidade de forma mais apurada (Riis et al., 2005; Stambor, 2005).

Riis et al. (2005) realizaram um estudo com um grupo de 49 pacientes que passavam por hemodiálise e um grupo de 49 pessoas saudáveis. Perceberam que as pessoas em hemodiálise costumavam se adaptar a sua condição e, embora relatassem a própria saúde como sendo pior do que a de pessoas saudáveis, elas não pareciam ser muito menos felizes do que aquelas que não tinham doença nos rins ou alguma outra alteração séria de saúde.

Ao mesmo tempo, as pessoas saudáveis são claramente inconscientes de que a adaptação à hemodiálise ocorre. Suas estimativas em relação ao bem-estar de pacientes em hemodiálise eram muito mais baixas do que esses pacientes realmente relatavam. Este estudo permitiu que se percebesse que as pessoas saudáveis subestimam a qualidade da experiência emocional da pessoa doente. Os pesquisadores notaram que os pacientes desenvolvem uma tendência a se focalizar mais nas experiências passadas positivas, sendo que esta pode ser uma parte crucial do processo da adaptação (Riis et al., 2005). 
Cada vez mais torna-se relevante avaliar a possível ligação entre bem-estar subjetivo e indicadores econômicos. É comum o pensamento de que maior riqueza estaria diretamente relacionada a maior felicidade. Porém, para Csikszentmihzlyi (1999), vantagens materiais não se traduzem em benefícios sociais e emocionais.

De acordo com Seligman (2004), existem muitas exceções à associação riqueza/satisfação, o que pode ser observado em países como Brasil, China e Argentina, onde se observou mais satisfação com a vida do que seria de se esperar em suas populações com sua riqueza. Países do antigo bloco soviético e o Japão, em contraponto, mostram-se menos satisfeitos do que se esperava encontrar.

Diener e Suh (1997) indicam que, muito embora desde a Segunda Guerra Mundial tenha havido um aumento de renda significativo em países como Estados Unidos, Japão e França, a avaliação do bem-estar subjetivo dessas populações permaneceu praticamente inalterada no período. Para Seligman (2004, p.69), o poder de compra e a satisfação com a vida tendem a seguir na mesma direção, mas a partir de uma determinada renda não se verifica a correlação entre aumento da riqueza e aumento do bem-estar subjetivo. Tendo em vista tal fato, a afirmação de Seligman (2004, p.72) de que "mais que o próprio dinheiro, o que influencia a felicidade é a importância que você dá a ele" parece condizente com a realidade.

Csikszentmihalyi (1999) afirma que, se as pessoas se esforçam para alcançar certa condição de riqueza pensando que isso as fará mais felizes, elas percebem que, alcançando tal condição, rapidamente se habituam a ela, a ponto de começarem a desejar um próximo padrão de riqueza. $O$ autor ainda relata que as pessoas costumam considerar suas posses não em termos do que precisam para viver com mais conforto, mas sempre se comparando com aqueles que têm mais do que elas. Assim, as pessoas relativamente ricas sentem-se pobres em comparação aos muito ricos, e a falta de felicidade é o resultado (Csikszentmihalyi, 1999).

Myers (2000) afirma que mesmo as pessoas muito ricas são apenas levemente mais felizes do que a média da população norte-americana. Para ele, mesmo tendo muito dinheiro, as pessoas concordam que ele pode aumentar ou diminuir a felicidade, dependendo de como é usado.
Diener e Seligman (2004) afirmam que o aumento de renda cria desejos materiais cada vez maiores. Dessa forma, com o passar do tempo, a mesma quantidade de renda que parecia ter resultados suficientes na satisfação de tais desejos resultaria em frustração, e consecutivamente, em menos bem-estar.

Além de indicadores econômicos, outro possível correlato do bem-estar subjetivo seriam os relacionamentos interpessoais e o próprio estado civil. Pesquisadores do bem-estar subjetivo acreditam que, sozinhos, os indicadores sociais não podem definir qualidade de vida (Diener \& Suh, 1997), embora alguns considerem que relações sociais positivas são necessárias para o bem-estar (Diener \& Selligman, 2004). Muitos estudos têm indicado que, comparadas a pessoas solteiras, as pessoas casadas têm melhor saúde física e psicológica, além de viverem mais (Burman \& Margolin, 1992).

Easterlin (2003) fez um estudo, que durou cerca de 10 anos, sobre a felicidade nos ciclos de vida, observando indivíduos com idade entre 18 e 19 anos na primeira autoavaliação, e entre 28 e 29 anos na segunda. Na faixa etária de 18 a 19 anos, quando a maioria de mulheres e homens ainda não se casou, a felicidade foi equivalente ao valor 2.1 em média (em uma escala de 1 a 3); nos 10 anos seguintes, aquelas que relataram estar casadas apresentaram uma medida de felicidade mais elevada (2.2 a 2.3 em média) do que aquelas que nunca se casaram (2.1 em média). Da mesma forma, casar-se novamente tem o mesmo efeito positivo na felicidade que uma primeira união.

O aumento do tempo de vida da população é um fato cada vez mais relevante para estudos que consideram a maneira como as pessoas envelhecem e sua qualidade de vida, o que torna a longevidade um importante correlato para o estudo do bem-estar subjetivo. Seligman (2004, p.75) indica que a visão de que gente jovem é mais feliz é equivocada. De acordo com a maturidade, os preditores de felicidade mudam, por exemplo, para pessoas com idade mais avançada, a satisfação proveniente das relações sociais e saúde se torna mais importante (Myers, 2000).

Danner, Snowdon e Friesen (2001), em seu estudo sobre a autobiografia de freiras realizado nos Estados Unidos, investigaram a possível associação da expressão emocional escrita com a longevidade. Foram analisadas as autobiografias escritas há 60 anos e as condições 
apresentadas pelas freiras na atualidade. Os autores investigaram as emoções positivas, negativas e neutras nos relatos. Todas as 180 autobiografias do estudo eram de freiras das cidades de Milwaukee, Wisconsin e Baltimore, que as escreveram entre os 18 e 32 anos de idade, e que no momento do estudo tinham entre $75 \mathrm{e}$ 95 anos. Todas elas tinham as mesmas histórias relacionadas a casamento e reprodução, tinham as mesmas atividades sociais, não fumavam nem bebiam quantidades excessivas de álcool e tinham status e ocupações sociais similares, além de acesso a cuidados médicos semelhantes. O estudo encontrou uma forte associação entre a emoção positiva nas autobiografias e a longevidade observada seis décadas depois. Esse estudo levanta questões sobre como a emoção positiva apresentada no início da vida pode estar relacionada à longevidade (Danner et al., 2001).

Baker, Cahalin, Gerste Burr (2005) defendem que o engajamento e o comprometimento em relação às atividades produtivas (físicas e sociais) em idade mais avançada são benéficos ao bem-estar subjetivo. Ainda, afirmam que a ampliação do suporte social aumentaria o bem-estar subjetivo.

Uma importante fonte de suporte social e possível correlato do bem-estar subjetivo seria a religiosidade. Procurando explicar essas associações entre a religiosidade e o bem-estar subjetivo, pesquisadores consideraram diversas possibilidades. Uma explicação parcial parece ser que as comunidades da fé fornecem sustentação social (Ellison, Gay \& Glass, 1989). A religião é praticada geralmente por meio de grupos de pessoas que se apoiam e que trocam experiências. Dessa forma, as relações sociais são favorecidas, e o sentimento de fazer parte de um grupo emerge.

Segundo Diener e Seligman (2004), o bem-estar é frequentemente associado à melhoria da produtividade do trabalhador e a relações interpessoais compensadoras. Myers (2000) acrescenta que outra possível explicação para a correlação entre religiosidade e bem-estar seria o fato de que a fé traz, para muitas pessoas, um senso de finalidade, um significado em sua vida.

\section{Medidas do bem-estar subjetivo}

Existem diferentes meios para a avaliação do 418 bem-estar subjetivo. Diener e Seligman (2004) conside- ram a importância e a necessidade de uma aproximação entre essas formas de medida.

Para Lyubomirsky e Lepper (1999), as medidas atuais do bem-estar subjetivo avaliam um de seus dois componentes (afetivo ou cognitivo). Pede-se aos respondentes que avaliem seus níveis de afeto positivo e negativo sobre um período de tempo, ou pede-se que façam um julgamento de sua qualidade total de vida.

As escalas de itens múltiplos são mais indicadas para a avaliação de mais de um componente do bem-estar subjetivo. Por outro lado, as de item único são vantajosas por serem curtas, mas existem muitas críticas quanto a seu uso. Não é possível avaliar a consistência interna dessas escalas, sendo a confiabilidade temporal a única medida para estimar sua confiabilidade. As respostas dadas tendem a se desviar para a categoria de maior felicidade, e os instrumentos falham em cobrir todos os aspectos do bem-estar subjetivo. O uso dessas escalas é mais indicado quando se pretende realizar uma medida breve acerca do bem-estar subjetivo, e elas devem ser usadas junto a outros instrumentos para que suas medidas sejam mais bem sustentadas e interpretadas (Diener, 1984).

\section{O Questionário de felicidade Oxford}

O Questionário de Felicidade Oxford (QFO) é um instrumento para medida do bem-estar subjetivo desenvolvido por Hills e Argyle (2002), que apresenta um total de 20 itens a serem respondidos em uma Escala Likert de seis pontos (Hills \& Argyle, 2002). Os autores indicam que a soma das pontuações dos itens é a medida de felicidade, com altas pontuações indicando maior felicidade.

Hills e Argyle (2002) encontraram que a consistência interna verificada no QFO indicou o valor $\alpha=0,91$. Os autores realizaram uma análise dos componentes principais e encontraram oito deles explicando 64,3\% da variância, mas, após realizarem uma Rotação Ortogonal (Varimax), encontraram itens similares em diferentes componentes, e uma minoria de itens com cargas mais ou menos iguais em dois ou mais componentes. Devido a essas circunstâncias, os autores consideraram que os fatores extraídos não poderiam ser corretamente interpretados. 
Para os autores, essa dificuldade de interpretação dos resultados referentes ao QFO poderia ser explicada como uma consequência da existência de um grande número de componentes extraídos a partir do critério denominado "Autovalor". Entretanto, realizada uma rotação oblíqua do questionário, não se identificou nenhum fator de segunda ordem. Ainda, uma reanálise dos oito componentes iniciais extraiu apenas um componente de segunda ordem, o que sugere que a medida do constructo do bem-estar subjetivo pode ser considerada unidimensional (Hills \& Argyle, 2002).

Kashdan (2004) apresenta um estudo no qual avalia o Questionário Oxford desenvolvido por Hills e Argyle (2002). O autor acredita que o QFO falha em discriminar entre o bem-estar subjetivo e as muitas outras forças humanas, que apresentam definições, teorias e medidas específicas. Ainda, os autores do instrumento não teriam fornecido dados suficientes para que os leitores pudessem avaliar os resultados obtidos de forma adequada. Ao invés disso, teriam considerado que a dificuldade de interpretação dos resultados poderia ser explicada como uma consequência do grande número de componentes extraídos por meio do método do Autovalor (Kashdan, 2004). Para o autor, seria equivocado o resultado da rotação oblíqua indicando a existência de um fator, principalmente se for considerado o resultado inconsistente sobre a análise dos fatores separados. Por fim, Kashdan (2004) afirma que o QFO parece ser um instrumento para medida de constructos difusos ao invés de um constructo claro, sendo que as relações entre o bem-estar subjetivo e diversos traços positivos podem mostrar-se maiores do que realmente são, o que poderia gerar dados equivocados em estudos subsequentes.

\section{A escala de felicidade subjetiva}

A Escala de Felicidade Subjetiva é um instrumento que foi desenvolvido por Lyubomirsky e Lepper (1999). Esse modelo de quatro itens é derivado de uma combinação inicial de 13 itens. Cada item deveria ser respondido dentro de uma Escala Likert de sete pontos. Segundo os autores, essa escala considera a felicidade da própria perspectiva do respondente. Analisando os dados obtidos, os autores não encontraram nenhuma diferença significativa para comparação entre sexos ou idades através da Escala de Felicidade Subjetiva. Os quatro itens que compõem a escala mostraram excelente consistência interna, sendo que o Alfa de Cronbach apresentou uma média igual a 0,86. A variabilidade teste-reteste variou de 0,55 a 0,90, tendo como média 0,72.

Para calcular o resultado, devem-se somar os números correspondentes às respostas e dividir por 8 . Nos Estados Unidos, a média para adultos é de 4,8, sendo que dois terços das pessoas ficam entre 3,8 e 5,8 (Seligman, 2004).

Kashdan (2004) considera que a Escala de Felicidade Subjetiva é um instrumento com boas propriedades psicométricas, que mede com a precisão aquilo a que se propõe e, ainda, que pode ser respondido em um curto período de tempo, o que, para o autor, é um aspecto positivo.

\section{Item de felicidade global}

O Item de Felicidade Global foi desenvolvido por Bradburn (1969) e consiste em uma única questão - "Considerando todas as coisas, quão feliz você está nos dias atuais?" - que deve ser respondida em uma Escala Likert de sete pontos, variando de "Não muito feliz" (correspondendo a 1) a "Muito feliz" (correspondendo a 7).

Esse é um item simples e de fácil compreensão. A utilização desse instrumento é relevante, ao permitir a medida da autoavaliação individual por intermédio da felicidade percebida no momento presente.

\section{Método}

A pesquisa foi realizada junto a 524 estudantes universitários voluntários da cidade de Ribeirão Preto, sendo 145 homens e 379 mulheres, com idade entre 17 e 54 anos ( $M=21,7, D P=4,4)$. Todos os participantes apresentavam o mesmo grau de instrução (ensino superior incompleto).

O material utilizado foi constituído de Instruções Gerais, Indicadores Sociodemográficos, Questionário de Felicidade Oxford, Escala de Felicidade Subjetiva e Item de Felicidade Global, apresentados de forma aleatória.

As aplicações foram feitas de forma coletiva. Cada estudante após assinatura do Termo de Consen- 
timento e Livre Esclarecimento recebia o material e era instruído a respondê-lo individualmente, sem que o tempo fosse cronometrado.

Esta pesquisa foi aprovada pelo Comitê de Ética em Pesquisa da Faculdade de Filosofia, Ciências e Letras da Universidade de São Paulo de Ribeirão Preto com emissão do parecer (CEP-FFCLRP no 189/2005), de acordo com a resolução 196/96 do Conselho Nacional de Saúde (MS).

\section{Resultados}

\section{Questionário de felicidade Oxford}

O cálculo da Correlação de Spearman entre os itens do QFO respondidos por participantes do sexo masculino indicou correlações significativas, mas baixas. O cálculo do coeficiente Alfa de Cronbach indicou um valor igual a 0,82 .

Na análise dos componentes principais foram extraídos onze componentes que responderam por $68,0 \%$ da variabilidade total. O primeiro componente respondeu por 19,7\% da variabilidade, e os outros por 8,4\%, 5,6\%, 5,1\%, 4,8\%, 4,6\%, 4,2\%, 4,2\%, 4,0\%, 3,6\% e 3,5\% respectivamente. Após a rotação, a variabilidade foi distribuída entre os componentes, que passaram a explicar de 10,6\% (Componente 1) a 4,5\% (Componente 11) da variabilidade total. Em seguida, foi calculada a matriz de correlação seguida pelo cálculo da matriz de correlação com a rotação Varimax (Tabela 1).

O cálculo da Correlação de Spearman entre os itens do QFO respondidos por participantes do sexo

Tabela 1. Consistência interna e variações nos valores após a retirada das questões de cada componente no Questionário Oxford para participantes do sexo masculino. Ribeirão Preto (SP), 2007.

\begin{tabular}{|c|c|c|c|c|c|}
\hline & Componente & $\alpha$ & & Itens & $\alpha$ se o item for deletado \\
\hline & \multirow[t]{6}{*}{1} & \multirow[t]{6}{*}{0,77} & 9 & Sinto-me sempre alegre e exaltado & 0,72 \\
\hline & & & 1 & Eu tenho sentimentos muito calorosos com quase todos & 0,75 \\
\hline & & & 26 & Eu sorrio muito & 0,72 \\
\hline & & & 14 & Sempre contagio de alegria outras pessoas & 0,74 \\
\hline & & & 2 & Sinto que possuo muita energia & 0,75 \\
\hline & & & 11 & Sempre tenho uma boa influência nos acontecimentos & 0,75 \\
\hline & \multirow[t]{4}{*}{2} & \multirow[t]{4}{*}{0,65} & 21 & Eu acho a maioria das coisas agradáveis & 0,52 \\
\hline & & & 23 & Sempre me saio bem em tudo que eu quero & 0,56 \\
\hline & & & 29 & Estou sempre comprometido e envolvido & 0,64 \\
\hline & & & 27 & Sinto-me capaz de conseguir qualquer coisa & 0,59 \\
\hline & \multirow[t]{3}{*}{3} & \multirow[t]{3}{*}{0,04} & 22 & Eu não acho que o mundo é um bom lugar & $-0,19$ \\
\hline & & & 13 & A vida é boa & $-0,46$ \\
\hline & & & 6 & Eu sou intensamente interessado em outras pessoas & 0,51 \\
\hline & \multirow[t]{3}{*}{4} & \multirow[t]{3}{*}{0,55} & 18 & Existe uma distância entre o que eu gostaria de fazer e o que faço & 0,52 \\
\hline & & & 8 & Estou muito satisfeito com tudo em minha vida & 0,30 \\
\hline D & & & 4 & Eu sou muito feliz & 0,50 \\
\hline$\widetilde{\Sigma}$ & \multirow[t]{2}{*}{5} & \multirow[t]{2}{*}{0,45} & 16 & Eu sinto que a vida é muito recompensadora & - \\
\hline 㿽 & & & 7 & Eu não me sinto particularmente satisfeito com a maneira que sou & - \\
\hline$\grave{\varsigma}$ & \multirow[t]{2}{*}{6} & \multirow[t]{2}{*}{0,40} & 24 & Eu não me acho atraente & - \\
\hline 艿 & & & 19 & Não acho fácil tomar decisões & - \\
\hline N & \multirow[t]{3}{*}{7} & \multirow[t]{3}{*}{0,51} & 10 & Não tenho particularmente um senso de significado e propósito para minha vida & 0,35 \\
\hline$\underline{\text { OO }}$ & & & 12 & Eu particularmente não sou otimista sobre o futuro & 0,33 \\
\hline $\bar{e}$ & & & 5 & Sinto que não estou, sobretudo, no controle de minha vida & 0,55 \\
\hline 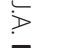 & \multirow[t]{2}{*}{8} & \multirow[t]{2}{*}{0,46} & 28 & Não me divirto com outras pessoas & - \\
\hline$D^{D}$ & & & 20 & Não me sinto particularmente saudável & - \\
\hline \multirow{2}{*}{$\overline{\underline{\Sigma}}$} & 9 & - & 15 & Sinto minha mente completamente alerta & - \\
\hline & 10 & - & 17 & Eu raramente sinto-me descansado & - \\
\hline & \multirow[t]{2}{*}{11} & \multirow[t]{2}{*}{$-0,36$} & 25 & Encontro beleza em algumas coisas & - \\
\hline & & & 3 & Não tenho particularmente memórias felizes do passado & - \\
\hline
\end{tabular}


feminino indicou correlações significativas, porém baixas. $O$ coeficiente Alfa de Cronbach foi adequado $(\alpha=0,84)$.

Na análise dos componentes principais, foram extraídos onze componentes respondendo por 68,0\% da variabilidade total do instrumento. $O$ primeiro componente respondia por $21,4 \%$ da variabilidade, e os outros por $7,3 \%, 5,5 \%, 4,3 \%, 4,0 \%, 3,9 \%, 3,8 \%$ e 3,7\% respectivamente. Após a rotação, a variabilidade foi distribuída entre os componentes, que passaram a explicar de 12,5\% (Componente 1) a 4,0\% (Componente 8) da variabilidade total. Seguindo a análise, foi calculada a matriz de correlação, seguida pelo cálculo da matriz de correlação com a rotação Varimax (Tabela 2).

Na análise de todos os participantes verificou-se consistência interna $(\alpha=0,83)$ adequada. Foram extraídos oito componentes respondendo por 52,8\% da variabilidade total, sendo que o primeiro respondeu por 20,7\%, e os outros componentes foram responsáveis por 7,2\%, 5,4\%, 4,2\%, 4,0\%, 3,7\%, 3,7\% e 3,6\% da variabilidade, respectivamente. Após a rotação realizada, a variabilidade foi distribuída de melhor forma entre os componentes, que passaram a explicar de 9,4\% (Componente 1) a 4,1\% (Componente 8) da variabilidade total.

Em seguida, foi realizada a decomposição da matriz de correlação, e esta matriz foi submetida à rotação Varimax. O valor obtido para o critério Kaiser-Meyer-Olkin foi igual a 0,86, indicando que a realização da análise fatorial foi apropriada para os dados em questão. Por intermédio da análise das comunalidades (Artes, 1998) percebeu-se que, com exceção da questão 5, que apresentou comunalidade de $29 \%$, todas as demais apre-

Tabela 2. Consistência interna e variações nos valores após a retirada das questões de cada componente no QFO para participantes do sexo feminino. Ribeirão Preto (SP), 2007.

\begin{tabular}{|c|c|c|c|c|}
\hline Componente & $\alpha$ & & $\alpha$ seo & em for deletado \\
\hline \multirow[t]{9}{*}{1} & \multirow[t]{9}{*}{0,81} & 9 & Sinto-me sempre alegre e exaltado & 0,77 \\
\hline & & 8 & Estou muito satisfeito com tudo em minha vida & 0,77 \\
\hline & & 16 & Eu sinto que a vida é muito recompensada & 0,79 \\
\hline & & 4 & Eu sou muito feliz & 0,79 \\
\hline & & 14 & Sempre contagio de alegria outras pessoas & 0,78 \\
\hline & & 13 & A vida é boa & 0,80 \\
\hline & & 26 & Eu sorrio muito & 0,80 \\
\hline & & 21 & Eu acho a maioria das coisas agradáveis & 0,79 \\
\hline & & 7 & Eu não me sinto particularmente satisfeito com a maneira que sou & 0,81 \\
\hline \multirow[t]{6}{*}{2} & \multirow[t]{6}{*}{0,68} & 3 & Não tenho particularmente memórias felizes do passado & 0,63 \\
\hline & & 10 & Não tenho particularmente um senso de significado e propósito para minha vida. & 0,63 \\
\hline & & 12 & Eu particularmente não sou otimista sobre o futuro & 0,62 \\
\hline & & 28 & Não me divirto com outras pessoas & 0,65 \\
\hline & & 22 & Eu não acho que o mundo é um bom lugar & 0,64 \\
\hline & & 24 & Eu não me acho atraente & 0,64 \\
\hline \multirow[t]{5}{*}{3} & \multirow[t]{5}{*}{0,66} & 27 & Sinto-me capaz de conseguir qualquer coisa & 0,56 \\
\hline & & 23 & Sempre me saio bem em tudo que eu quero & 0,58 \\
\hline & & 11 & Sempre tenho uma boa influência nos acontecimentos & 0,62 \\
\hline & & 2 & Sinto que possuo muita energia & 0,61 \\
\hline & & 18 & Existe uma distância entre o que eu gostaria de fazer e o que faço & 0,66 \\
\hline \multirow[t]{2}{*}{4} & \multirow[t]{2}{*}{0,35} & 1 & Eu tenho sentimentos muito calorosos com quase todos & - \\
\hline & & 6 & Eu sou intensamente interessado em outras pessoas & - \\
\hline \multirow[t]{3}{*}{5} & \multirow[t]{3}{*}{0,40} & 17 & Eu raramente sinto-me descansado & 0,32 \\
\hline & & 20 & Não me sinto particularmente saudável & 0,26 \\
\hline & & 5 & Sinto que não estou, sobretudo, no controle de minha vida & 0,35 \\
\hline \multirow[t]{2}{*}{6} & \multirow[t]{2}{*}{$-0,27$} & 19 & Não acho fácil tomar decisões & - \\
\hline & & 15 & Sinto minha mente completamente alerta & - \\
\hline 7 & - & 29 & Estou sempre comprometido e envolvido & - \\
\hline 8 & - & 25 & Encontro beleza em algumas coisas & - \\
\hline
\end{tabular}


sentaram valores que explicavam a variabilidade dos dados. O item mais bem explicado pelos componentes foi o item 25 (com 75\%). Posteriormente, verificou-se a consistência dos oito componentes extraídos, e os mesmos foram nomeados de acordo com as questões que os compunham (Tabela 3).

\section{Escala de felicidade subjetiva}

Inicialmente realizou-se a análise dos componentes principais a partir das respostas dos par- ticipantes do sexo masculino, cuja mediana foi 5,5 .

O cálculo do coeficiente Alfa de Cronbach indicou uma consistência interna $(0,74)$ adequada. A análise dos componentes principais trouxe como resultado a existência de apenas um componente com autovalor inicial acima de 1,00 para participantes do sexo masculino. Esse componente, sozinho, respondeu por 59,7\% da variância total. A matriz de correlação dos componentes também confirmou a existência de um único componente nesse instrumento.

Tabela 3.Valores do Alfa de Cronbach para cada componente e variações nos valores após a retirada das questões que compõem cada fator no QFO. Ribeirão Preto (SP), 2007.

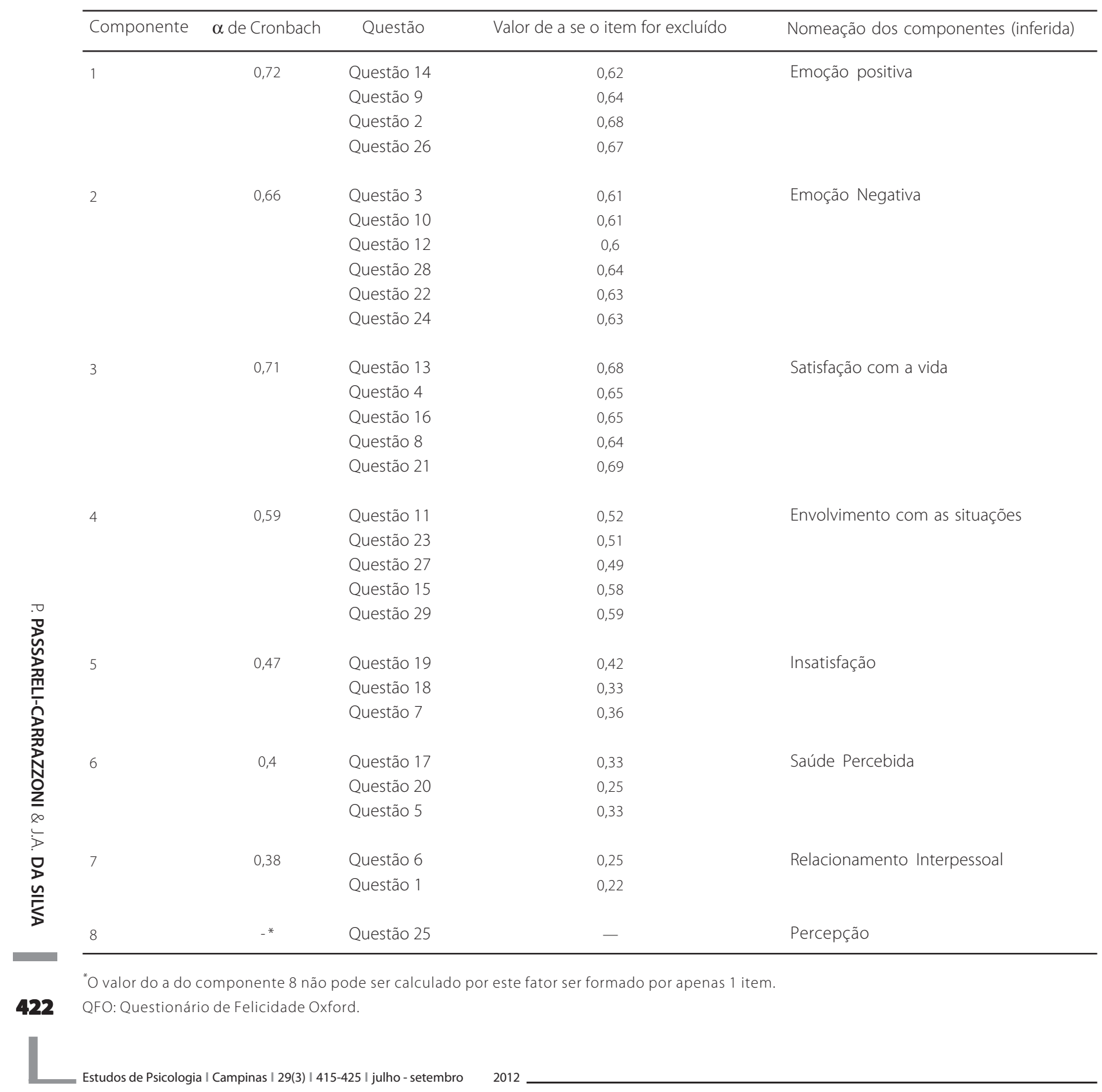


Na análise dos componentes principais a partir das respostas de participantes do sexo feminino, a mediana foi igual a 6 . O cálculo do coeficiente Alfa de Cronbach indicou consistência interna de 0,75. A análise dos componentes principais trouxe como resultado a existência de apenas um componente com autovalor inicial acima de 1,00. Esse componente, sozinho, respondeu por 59,7\% da variância total. A matriz de correlação dos componentes confirmou a existência de um único componente.

Avaliando os resultados de todos os participantes de forma conjunta, percebe-se que a maior parte das respostas concentrou-se nos valores 5, 6 e 7 da Escala Likert. A mediana das respostas para todos os participantes foi igual a 6 . O cálculo do coeficiente Alfa de Cronbach indicou consistência interna $(0,75)$ adequada. A análise dos componentes principais trouxe como resultado a existência de apenas um componente com autovalor inicial acima de 1,00, sendo que esse componente respondeu por 59,7\% da variância total. A matriz de correlação dos componentes também confirmou a existência de um único componente nesse instrumento.

\section{Item de felicidade global}

A mediana das respostas dos participantes do sexo masculino, dos participantes do sexo feminino, e do conjunto total de participantes para o Item de Felicidade Global foi igual a 6.

\section{Discussão}

O Questionário de Felicidade de Oxford mostrou consistência interna para todos os grupos avaliados (Pasquali, 1999), porém inferior aos do instrumento original (Hills \& Argyle, 2002). Esse fato indica que o instrumento mede adequadamente aquilo a que se propõe. Porém, a partir das críticas apresentadas por Kashdan (2004), foi realizada uma análise mais profunda para avaliação dos itens.

O Questionário de Felicidade de Oxford apresentou um total de onze componentes para o grupo do sexo masculino, o que divergiu tanto do grupo do sexo feminino, como do grupo total de participantes e do questionário original (que apresentaram oito com- ponentes). Esse fato parece indicar que o instrumento não apresenta estabilidade de conteúdo. Ainda, mesmo apresentando o mesmo número de componentes, os itens que formavam cada componente não foram os mesmos para o grupo do sexo feminino e para o grupo total.

A partir da análise dos componentes obtidos para os participantes do sexo masculino e dos itens que os compunham, notou-se que não ocorreu um agrupamento dos itens que representam o afeto positivo em um único componente, e sim um agrupamento que resultou em um número maior de componentes. Por exemplo, os itens 8 e 4, que representam afeto positivo, agruparam-se ao item 18, que representa autonomia e formaram o componente 4. Porém, mesmo sendo agrupados de forma difusa, percebe-se que os componentes descrevem claramente seu significado.

Ainda, pode-se afirmar que os componentes resultantes da Análise dos Componentes Principais são adequados e consistentes. Os valores de Alfa de Cronbach $(0,45$ a 0,76) são adequados (Cronbach, 1996), porém apenas o Componente 1 apresenta $\alpha$ superior a 0,70 (Pasquali, 1999).

Quando verificado o valor do $\alpha$ de cada componente se os itens fossem excluídos, percebe-se que, com exceção dos itens 15 e 17, que sozinhos representam um componente cada, todos os outros representariam diminuição dos valores de $\alpha$, ou seja, todos são relevantes para os componentes dos quais fazem parte. Mesmo os itens 15 e 17, embora sendo os únicos constituintes de seus Componentes ( 9 e 10, respectivamente), têm cargas elevadas $(0,81$ e 0,84), o que indica sua relevância.

A análise dos componentes obtidos para os participantes do sexo feminino indica uma tendência em agrupar os itens a partir de seu real significado. Por exemplo, todos os itens relativos a afeto positivo e humor foram agrupados no Componente 1. Por outro lado, os itens relacionados ao afeto negativo, incluindo autoestima(-), sendo de propósito(-) e envolvimento social(-) foram agrupados no Componente 2 .

Os valores de Alfa de Cronbach são adequados (Cronbach, 1996), porém, da mesma forma como é observado no caso de participantes do sexo masculino, apenas o Componente 1 apresenta $\alpha$ superior a 0,70 (Pasquali, 1999). Quando verificado o valor do $\alpha$ de cada 
componente se os itens fossem excluídos, percebe-se que os itens 19 e 29, mesmo sendo os únicos representantes dos Componentes 6 e 7, respectivamente, apresentam cargas elevadas $(0,72$ e 0,77), o que sugere sua relevância.

Conclui-se, então, que a maneira como os participantes do sexo masculino responderam às questões do instrumento pode representar uma maneira divergente da feminina de avaliar seu bem-estar subjetivo, mas que mesmo sendo divergente leva a um resultado muito próximo, pois as medianas apresentadas para ambos os sexos são iguais. Por se tratar de um instrumento para a medida de um aspecto subjetivo, pode-se inferir que as diferenças na maneira como homens e mulheres avaliam seu bem-estar subjetivo é resultado da própria diferença entre os sexos em perceber seu bem-estar individual, e quais são os caminhos que levam a ele.

A análise do QFO respondido por todos os participantes traz resultados indicando que a predominância de participantes do sexo feminino tende a dominar a amostragem geral. É interessante perceber que os resultados dessa amostra são semelhantes aos alcançados pelos autores do artigo original (Hills \& Argyje, 2002), o que sugere que o número superior de mulheres participantes de seu estudo causou o mesmo efeito no resultado final.

A análise da Escala de Felicidade Subjetiva indica a existência de um único componente respondendo por mais de 59\% da variância acumulada para participantes do sexo masculino, feminino e para o grupo total de participantes. Tais resultados corroboram os estudos de Lyubomirsky e Lepper (1999). O Alfa de Cronbach calculado a partir das respostas dadas por participantes do sexo masculino e do feminino $(0,74$ e 0,75 respectivamente) mostra-se inferior ao do instrumento original $(\alpha=0,86)$, porém adequado (Cronbach, 1996; Pasquali, 1999).

Os resultados apresentados confirmam os dados de Lyubomirsky e Lepper (1999) e as observações de Kashdan (2004), que considera a Escala de Felicidade Subjetiva como um instrumento com boas propriedades psicométricas e preciso na medição daquilo a que 424 se propõe. Dessa forma, verifica-se a consistência e ade- quação desse instrumento para a medida do bem-estar subjetivo.

Na análise do item de Felicidade Global, percebe-se que homens e mulheres apresentaram medidas iguais de bem-estar subjetivo. A tendência das respostas tanto de homens como de mulheres concentra-se nos valores mais elevados da Escala Likert, sendo que na análise da frequência relativa de respostas nota-se uma distribuição semelhante para todos os grupos de participantes. Esses resultados são semelhantes àqueles apresentados pelos outros instrumentos utilizados, e confirmam a observação de Diener (1984) de que escalas curtas devem ser usadas junto a outros instrumentos para que suas medidas sejam mais bem sustentadas e interpretadas.

A análise dos três instrumentos de avaliação do bem-estar subjetivo indicou que eles são boas medidas de avaliação. Embora não tenha sido objetivo deste estudo realizar a validação dos mesmos, os resultados obtidos indicaram sua qualidade psicométrica.

\section{Considerações Finais}

Por todos os resultados alcançados e pela crescente atenção que tem sido dada à Psicologia Positiva, pode-se afirmar que a continuidade de estudos envolvendo o tema é relevante. O mesmo pode ser dito a respeito de avaliações sobre o bem-estar subjetivo, embora seja importante considerar a escolha de bons instrumentos de avaliação como o primeiro passo, devido à subjetividade do tema.

Os três instrumentos escolhidos para este estudo mostraram-se adequados, embora se deva ressaltar que estudos psicométricos visando à validação dos mesmos para o uso junto à população brasileira devam ser realizados. Ainda, os resultados obtidos indicaram a importância de se utilizar mais de um instrumento quando se pretende investigar temas como o bem-estar subjetivo, o que possibilita uma visão mais completa sobre diferentes aspectos de um mesmo tema.

Por fim, conclui-se que atenção especial deve ser dada aos correlatos do bem-estar subjetivo. Estudos envolvendo condições de saúde/doença, renda, escolaridade, diferenças culturais, entre outros correlatos, podem trazer importantes contribuições para a Psicologia. 


\section{Referências}

Baker, L. A., Cahalin, L. P., Gerst, K., \& Burr, J. A. (2005). Productive activities and subjective well-being among older adults: the influence of number of activities and time commitment. Social Indicators Research, 73 (3), 431-458.

Bradburn, N. M. (1969). The structure of psychological well-being. Chicago: Aldine.

Burman, B., \& Margolin, G. (1992). Analysis of the association between marital relationships and health problems: an interactional perspective. Psychological Bulletin, 112 (1), 39-63.

Cronbach, L. J. (1996). Fundamentos da testagem psicológica (5a ed.). Porto Alegre: Artes Médicas.

Csikszentmihalyi, M. (1999). If we are so rich, why aren't we happy? American Psychologist, 54 (10), 821-827.

Danner, D. D., Snowdon, D. A., \& Friesen, W. V. (2001). Positive emotions in early life and longevity: findings from the nun study. Personality Processes and Individual Differences, 80 (5), 804-813.

Diener, E. (1984). Subjective well-being. Psychological Bulletin, 95 (3), 542-575.

Diener, E. (2000). Subjective Well-Being: the science of happiness and a proposal for a national index. American Psychologist, 55 (1), 34-43.

Diener, E., \& Seligman, M. E. P. (2002). Very happy people. Psychological Science, 13 (1), 82-84.

Diener, E., \& Seligman, M. E. P. (2004). Beyond money: toward an economy of well-being. Psychological Science in the Public Interest, 5 (1), 1-31.

Diener, E., \& Suh, E. (1997). Measuring quality of life: economic, social, and subjective indicators. Social Indicators Research, 40, 189-216.

Diener, E., Suh, E. M. R., Lucas, R. E., \& Smith, H. (1999). Subjective well-being: three decades of progress. Psychological Bulletin, 125 (2), 276-302.

Easterlin, R. A. (2003). Explaining happiness. PNAS, 100 (10), 11176-11183.

Ellison, C. G., Gay, D. A., \& Glass, T. A. (1989). Does religious commitment contribute to individual life satisfaction? Social Forces, 68 (1), 100-123.
Fredrickson, B. L. (1998). What good are positive emotions? Review of General Psychology, 2 (3), 300-319.

Hills, P., \& Argyle, M. (2002). The Oxford Happiness Questionnaire: a compact scale for the measurement of psychological well-being. Personality and Individual Differences, 33 (7), 1073-1082.

Kashdan, T. B. (2004). The Oxford Happiness Questionnaire: a compact scale for the measurement of psychological well-being. Personality and Individual Differences, 33 (7), 1071-1082.

Lyubomirsky, S., \& Lepper, H. S. A. (1999). Measure of subjective happiness: preliminary reliability and construct validation. Social Indicators Research, 46 (2), 137-155.

Myers, D. G. (2000). The funds, friends, and faith of happy people. American Psychologist, 55 (1), 56-67.

Pasquali, L. (1999). Instrumentos psicológicos: manual prático de elaboração. Brasília: LabPAM.

Passareli, P. M., \& Da Silva, J. A. (2007). Psicologia positiva e o estudo do bem-estar subjetivo. Estudos de Psicologia (Campinas), 24 (4), 513-517. doi: 10.1590/S0103-166X20 0700040010.

Riis, J., Loewenstein, G., Baron, J., Jepson, C., Fagerlin, A., \& Ubel, P. A. (2005). Ignorance of hedonic adaptation to hemo-dialysis: a study using ecological momentary assessment. Journal of Experimental Psychology: General, 134 (1), 3-9.

Salovey, P., Rothman, A. J., Detweiler, J. B., \& Steward, W. T. (2000). Emotional states and physical health. American Psychologist, 55 (1), 110-121.

Seligman, M. E. P. (2004). Felicidade autêntica: usando a nova psicologia positiva para a realização permanente. Rio de Janeiro: Objetiva.

Seligman, M. E. P., \& Csikszentmihalyi, M. (2000). Positive psychology: an introduction. American Psychologist, 55 (1), 5-14.

Stambor, Z. (2005). People in poor health are happier than others think. American Psychology Association, 36 (4), 13.

Steptoe, A., Wardle, J., \& Marmot, M. (2005). Positive affect and health-related neuroendocrine, cardiovascular, and inflammatory processes. PNAS, 102 (18), 6508-6512.

Recebido em: 16/9/2009

Versão final reapresentada em: 3/2/2012

Aprovado em: 10/2/2012 
